\title{
Inhibition of embryonic development by microcystin-LR in zebrafish, Danio Rerio
}

\author{
Pei-Jen Wang ${ }^{\mathrm{a}}$, Ming-Shan Chien ${ }^{\mathrm{b}}$, Fong-June $\mathrm{Wu}^{\mathrm{a}}$, \\ Hong-Nong Chou ${ }^{\mathrm{a}, \mathrm{c}}$, Shyh-Jye Lee $\mathrm{e}^{\mathrm{b}, \mathrm{c}, *}$ \\ ${ }^{a}$ Institute of Fisheries Science, National Taiwan University, Taipei, Taiwan, ROC \\ ${ }^{\mathrm{b}}$ Institute of Zoology, National Taiwan University, Taipei, Taiwan, ROC \\ ${ }^{\mathrm{c}}$ Department of Life Sciences, National Taiwan University, Taipei, Taiwan, ROC
}

Received 7 September 2004; revised 22 October 2004; accepted 25 October 2004

Available online 10 December 2004

\begin{abstract}
Microcystin-LR (MC-LR), a cyanobacterial toxin, is a potent inhibitor of protein phosphatase 1 (PP1) and protein phosphatase 2A (PP2A). PP1 and PP2A are critical regulators in embryonic development. However, the effects of MC-LR in embryonic development have been controversial. MC-LR has been demonstrated to be highly toxic in medaka, but not in zebrafish or rabbit embryos. The causes of difference may be due to membrane impermeability that impaired the delivery of MC-LR into cytoplasm of zebrafish and rabbit embryos. Therefore, we microinjected MC-LR directly into developing zebrafish embryos and investigated the effects of MC-LR on embryonic development. We demonstrated that MC-LR induced the lethality of zebrafish embryos in a dose- and time-dependent manner. MC-LR also induced the loss of blastomere coherence via the interference of $\beta$-catenin and cadherins distributions. Furthermore, the MC-LR treated fry revealed various developmental defects. These results suggested that MC-LR might affect the phosphorylation equilibrium of signaling molecules, including $\beta$-catenin and cadherins, required early in zebrafish embryonic development.
\end{abstract}

(C) 2004 Elsevier Ltd. All rights reserved.

Keywords: Microcystin; PP1; PP2A; Zebrafish; Embryos; $\beta$-catenin and cadherin

\section{Introduction}

The toxicity of MCs has been associated with fish killed worldwide (Watanabe et al., 1996). Among the fish species killed by MCs, cyprinids are most affected, and to a less extent to the others (Fischer and Dietrich, 2000). By analyzing the diets of affected species, it appears that the toxicity of MCs depends on the amount of toxin ingested. However, by gavaging a bolus dose of MC-LR containing

\footnotetext{
* Corresponding author. Address: 1 Roosevelt Road, Section 4, Institute of Zoology, National Taiwan University, 209 Fisheries Science Building, Taipei, Taiwan 106, ROC. Tel.: +88612 33665902; fax: +8861233662457.

E-mail address: jefflee@ntu.edu.tw (S.-J. Lee).
}

lyophilized cyanobacteria to carp or rainbow trout, it appears that carp is more susceptible to MC-LR compared to rainbow trout (Tencalla et al., 1994; Tencalla and Dietrich, 1997). Therefore, the MC-LR gavaging experiments appeared not to be consistent to serve as a bioassay system in fish.

MC-LR is a potent inhibitor of serine/threonine protein phosphatase 1 and $2 \mathrm{~A}$ ( $\mathrm{PP} 1$ and $\mathrm{PP} 2 \mathrm{~A}$ ) with an $\mathrm{IC}_{50}$ of about 0.1-1.0 nM (Honkanen et al., 1990). The inhibition of PP1 and PP2A results in hyperphosphorylation of proteins, which may be responsible for the toxicity of microcystins. During embryonic development, the equilibrium of phosphorylation and dephosphorylation status of signaling molecules is tightly regulated for the control of rapid cleavages and cellular differentiation. Dynamic changes in cytoskeleton play a major role in embryo 
reorganization. Microcystins have been demonstrated to impair cytosleleton by hyperphosphorylation of the major intermediate proteins keratin 8 and 18 in a dose- and timedependent manner (Ohta et al., 1992; Toivola et al., 1997). Microcystins have also been reported to perturb the functions of motor protein dynein (Runnegar et al., 1997) and tubulin (Ding et al., 2000). It is therefore reasonable to hypothesize that MC-LR should disrupt early embryonic development by interfering with the cytoskeletal dynamic within the embryo. A couple of attempts have been reported to evaluate the effects of MC-LR on early embryonic development, including rabbit (Frangez et al., 2003) and zebrafish (Oberemm et al., 1997, 1999) studies. However, both studies revealed no significant defects of early embryos treated with MC-LR. These results are clearly against our hypothesis and the well established PP1 and PP2A blocking ability by MC-LR. In both investigations, the membrane-intact embryos were used. It was very likely that the membrane barrier precluded MCLR from entering cytoplasm. However, Wiegand et al. (1999), have shown that there was a detectable uptake of C-14 labeled MC-LR by early zebrafish embryos and the lack of toxicity may due to the activities of detoxication enzymes like glutathione S-transferase (GST). C-14 labeled MC-LR uptake reached $0.44 \mathrm{ng}$ per egg, which seems to be high enough to exert MC-LR's functions. However, since the C-14 labeled MC-LR uptake was measured using the whole egg (the embryo itself, the chorion, and the perivitelline fluid), one could not exclude the possibility that MC-LR was trapped mainly in the chorion and perivitelline fluid. Therefore, instead of relying on cellular uptake of MC-LR, we took a more direct approach by microinjecting MC-LR into zebrafish embryos to bypass membrane barriers. We found that (1) MC-LR dose- and time-dependently induced embryonic malformation and death. (2) MC-LR caused the loss of blastomere coherence by disrupting the distributions of $\beta$-catenin and cadherin.

\section{Materials and methods}

\subsection{Fish Husbandry and embryo collection}

Zebrafish, Danio rerio, were raised on a 14-h day/10-h night cycle at $26^{\circ} \mathrm{C}$. Eggs were collected at 15-20 min intervals after spawning, washed and incubated in Ringer's solution $\left(116 \mathrm{mM} \mathrm{NaCl}, 2.9 \mathrm{mM} \mathrm{KCl}, 1.8 \mathrm{mM} \mathrm{CaCl}_{2}\right.$ and $5 \mathrm{mM}$ HEPES) at $28.5^{\circ} \mathrm{C}$ until use. All chemicals were from Sigma (St Louis, MO) unless otherwise stated.

\subsection{Purification of $M C-L R$}

MC-LR was extracted and purified from Microcystin aeruginosa as described in detail by Lee et al. (1998).

\subsection{Microinjection procedures}

Glass capillaries of $1.14 \mathrm{~mm}$ O.D. and $0.5 \mathrm{~mm}$ I.D. (World Precision Instrument Inc., Sarasota, FL) were pulled using a horizontal puller (P-97, Sutter Instrument, Navato, CA). Embryos at desired stages as judged according to Kimmel et al. (1995) were immobilized at an injection trough on a $100 \mathrm{~mm} 2 \%$ agar plate. MC-LR was dissolved in injection buffer $(68.5 \mathrm{mM} \mathrm{NaCl}, 1.35 \mathrm{mM} \mathrm{KCl}, 5 \mathrm{mM}$ $\mathrm{Na}_{2} \mathrm{HPO}_{4}$ and $1 \mathrm{mM} \mathrm{KH}_{2} \mathrm{PO}_{4}$ and $0.25 \%$ (w/v) phenol red) with different concentrations of MC-LR as indicated. An injection pipette was forced into the chorion and the yolk cell to reach the junction between yolk cell and blastodisc where $2.3 \mathrm{~nL}$ of solution was ejected by using a nanoliter injector (World Precision Instrument Inc., Sarasota, FL). After injection, embryos were recovered from injection troughs and cultured in Ringer's solution at $28.5^{\circ} \mathrm{C}$ until examined.

\subsection{Whole-mount immunocytochemistry and confocal microscopy}

Zebrafish embryos at designated stages were fixed in $4 \%$ paraformaldehyde in PBS $(137 \mathrm{mM} \mathrm{NaCl}, 2.7 \mathrm{mM} \mathrm{KCl}$, $10 \mathrm{mM} \mathrm{Na}_{2} \mathrm{HPO}_{4}$ and $2 \mathrm{mM} \mathrm{KH}_{2} \mathrm{PO}_{4}$ ) overnight at $4{ }^{\circ} \mathrm{C}$, washed in PBS and manually dechorionated. Twenty embryos were incubated with a polyclonal anti- $\beta$-catenin antibody (Sigma C2206, diluted at 1:400) or a polyclonal anti-pan cadherin antibody (Sigma C3678, diluted at 1:100) with continuous rotation at $4{ }^{\circ} \mathrm{C}$ overnight. After four washes in PBS containing $0.5 \%$ Triton X-100 (PBT), embryos were incubated in a 1:250 or 1:500 dilution of a FITC-conjugated goat anti-rabbit antibody (\#111-095-144, Jackson ImmunoResearch Laboratories, West Grove, PA) for anti- $\beta$-catenin or anti-pan cadherin antibody-labeled embryos, respectively, at $4{ }^{\circ} \mathrm{C}$ overnight. Embryos were transferred to $30 \%$ glycerol first and then $50 \%$ glycerol for confocal microscopic observations at the Center of TwoPhoton Laser Confocal Microscope, National Taiwan University. To reveal the nuclei, embryos were washed in PBT and then incubated in $10 \mu \mathrm{g} / \mathrm{mL}$ Hoechst dye for 30 mins at room temperature before examination. Unbound Hoechst dye was removed by washing in PBT and examined under an epifluorecent microscope.

\subsection{Embryo observations and photography}

Observation of embryonic development was made at designated time under a Leica Mz75 stereomicroscope, a Leica DMIRB fluorescent microscope, or a Leica TCS SP2 confocal microscope equipped with an $\mathrm{Ar}-\mathrm{Kr}$ two photon laser system located. All photographs were taken by a Nikon digital camera and analyzed using Adobe Photoshop 7.0 except for the confocal images, which were photographed and edited utilizing the Leica Confocal Software. 


\subsection{Statistical analysis}

Experimental values are expressed as means \pm standard error of means and analyzed in Excel using Two-Factor ANOVA with Replication.

\section{Results}

To study the toxicity of MC-LR in zebrafish, we microinjected MC-LR into 2-4 cell stage embryos. Within half an hour after injection of microcystin-LR (MC-LR), embryos showed signs of malformation. The most notable change in the MC-LR-injected embryos was the loss of coherence of blastomeres. A normal zebrafish embryo has a tightly adhered blastomeres arranged in tiers as shown in Fig. 1A. In contrast, MC-LR induced the deterioration of embryos by blocking the adherence of blastomeres (Fig. 1C). We were also interested to know if MC-LR affected nuclear division, since cell cycle progression is regulated by phosphorylation and dephosphorylation. In the controlinjected embryo all nuclei were clearly visible by Hoechst dye staining except one nucleus was out of focus as shown in Fig. 1B. In contrast, we observed only two nuclei in an 8-cell stage embryo treated with $750 \mathrm{nM}$ MC-LR in one focal plane
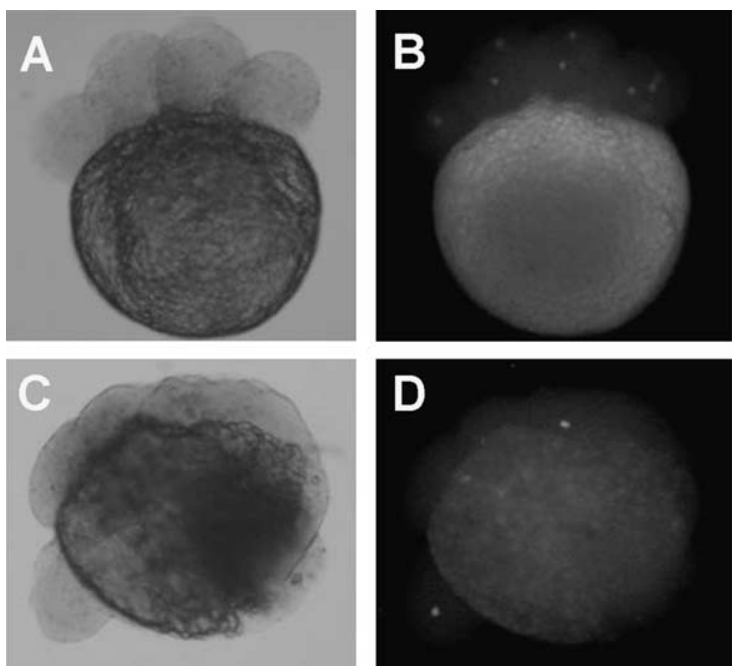

Fig. 1. MC-LR causes the loss of adherence of blastomeres in zebrafish embryos. After the injection of $900 \mathrm{nM} \mathrm{MC-LR,} \mathrm{a}$ zebrafish embryo could still divide, but the blastomeres lost compact adherence to each other (C) as compared to the controlinjected embryos (A). Blastomeres of the control-injected 8-cell embryos were nicely arranged into two tiers with nuclei clearly stained by Hoechst dye in each blastomere (B). One nucleus is out of focus in panel B. The nuclear cleavage appears not normal in MC-LR injected embryo at the first glance, since only 2 nuclei are visible on the focal plan shown in panel D. However, it was due to the irregular arrangement of blastomeres that we were able to locate all 8 nuclei in the 8 -cell stage embryo by scanning through different focal planes.

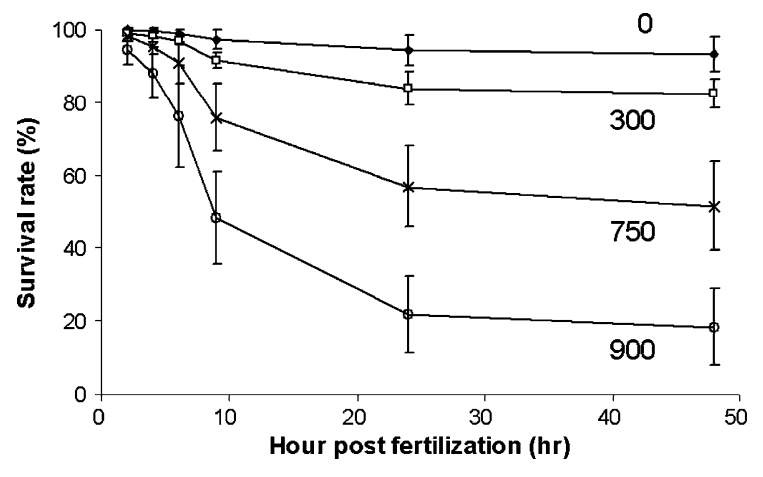

Fig. 2. MC-LR causes gradual death of zebrafish embryos in a dosedependent manner. An hour post injection with MC-LR at indicated amounts in $\mathrm{nM}$ gradual death occurred and the embryos survival rate continued to decrease until $24 \mathrm{~h}$ post injection. Fertilized eggs were injected at the $2-4$ cell stage and cultured in Ringer's solution at $28.5^{\circ} \mathrm{C}$. Embryos were examined at the designated time and the white/opaque embryos were scored as dead. These results were cumulated data of five independent experiments.

shown in Fig. 1D. However, by examining through different focal planes, we were able to find all 8 nuclei in the embryo treated with MC-LR (data not shown). Therefore, MC-LR appeared not interfering with the karyokinesis. Although, MCLR-treated embryos could still develop, but they were gradually dying and embryos turned opaque and disintegrating. To analyze the dose-dependent lethality of MC-LR, we injected embryos with different amounts of MC-LR. Injecting embryos after the 1-cell stage allowed us to remove easily unfertilized eggs from our statistics. We then examined survival rates of embryos at designated time points (Fig. 2). As shown in Fig. 2, 93.2 $\pm 4.8 \%(N=5)$ of 311 control-injected embryos survived at $48 \mathrm{~h}$ post injection. As MC-LR was injected, the survival rates of embryos decreased with the increase in time of culture post fertilization and the elevation of intracellular MC-CR concentration from 300 to $900 \mathrm{nM}$. At the end of examination ( $48 \mathrm{~h}$ post fertilization), embryonic survival rate decreased significantly to $82.5 \pm 3.9 \%$ of 319 embryos, $51.5 \pm 12.1 \%$ of 353 embryos and $18.5 \pm 10.3 \%$ of 343 embryos injected with 300, 750 and $900 \mathrm{nM}$ MC-LR, respectively, in 5 independent trials. Using the Two-Factor Analysis of Variance, the effects of intracellular MC-LR concentration and the time of culture post fertilization on embryonic survival were analyzed. Both factors and their interaction significantly ( $p \leq 4.65 \mathrm{E}-23)$ affected the survival rate of MC-LR-injected embryos. Thus, MC-LR induced embryonic death in a dose- and time-dependent manner. For those MC-LR-treated fry survived at $48 \mathrm{~h}$ post injection, various defects were shown in Fig. 3. The most frequent observed defects appeared in tails. Tails were curving (Fig. 3A), bent or twisting (Fig. 3B). In some cases, cyclopia occurred while a residual eye was still visible (arrow) as shown in Fig. 3C. Fig. 3D shows a fry with edema in pericardial sac (PS) and hatching gland (HG). Some of those fry with edema could survive up to 6-7 days post fertilization, but the edema in 

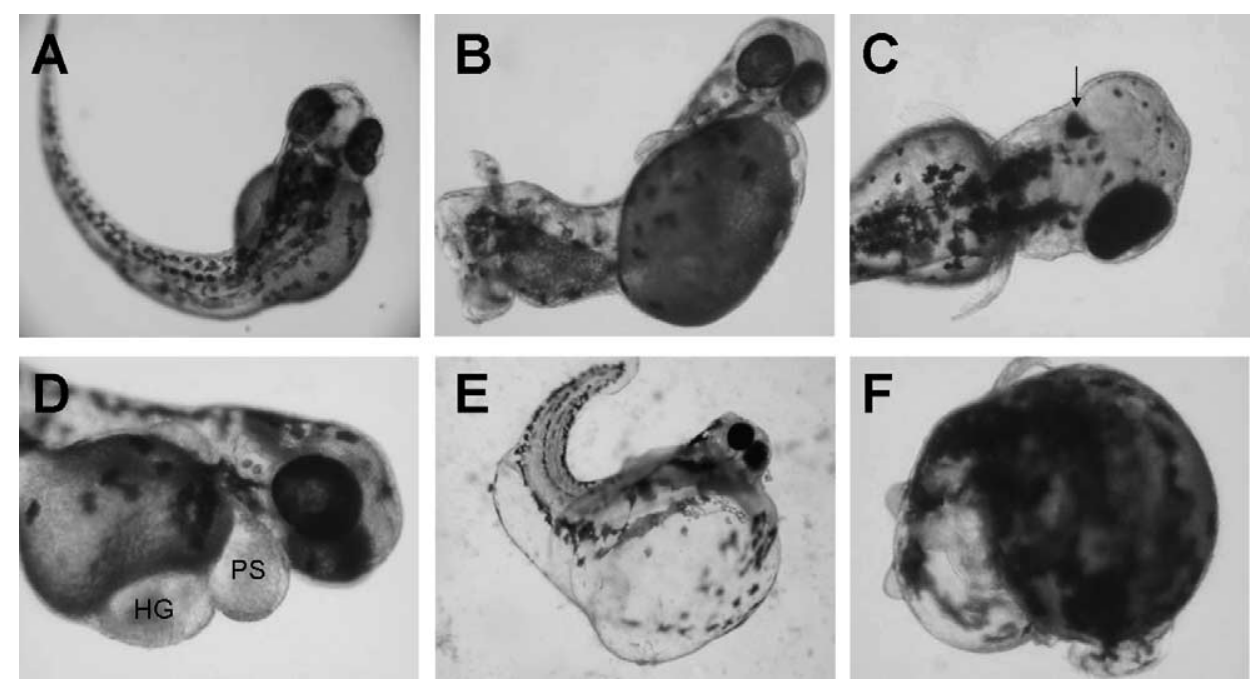

Fig. 3. MC-LR induces different developmental defects in zebrafish fry. MC-LR-treated embryos were cultured in Ringer's solution for $48 \mathrm{~h}$ and photographed except (E), which was taken at 6 days post fertilization. Various defects were detected in MC-LR-treated embryos, including trunk and tail curving (A), bent or twisting tails (B), cyclopia whose residual eye was indicated by an arrow (C), edema in pericardial sac (PS) and hatching gland (HG) (D), curving, rounding, deteriorated and eventually dying (F). Edema in pericardial sac and hatching gland could be worse where the curving of the trunk occurred if they survived in this case 6 days post fertilization (E).

pericardial sac and hatching gland was worse that a curvature of trunk occurred (Fig. 3E). In some severe cases, the fry was curving, rounding, deteriorating and eventually dying (Fig. $3 F)$. Due to the broad spectrum of defect patterns, we assume MC-LR perturbed the phosphorylation status of some key enzymes, which might interfere with downstream molecules required for proper embryonic development and subsequent organogenesis.

Blocking of blastomeres coherence by MC-LR (Fig. 1) is an intriguing observation. Since E-cadherin has been shown to mediate the blastomere adherence via $\beta$-catenin (Gotz et al., 2000), we investigated whether MC-LR inhibits blastomeres coherence by disturbing $\beta$-catenin/cadherin signaling by examining the distributions of $\beta$-catenin and cadherins in embryos treated with or without MC-LR. In Fig. 4, we show confocal photographs of a single section of $3 \mu \mathrm{m}$ (Fig. 4A, B, E, F) or 3D stacking graphs of 20 sections (Fig. 4C, D, G, H) for embryos treated in the presence (Fig. 4B, D, F, H) or absence (Fig. 4A, C, E, G) of 900 nM MC-LR. Both $\beta$-catenin (Fig. 4A, C) and cadherins (Fig. 4E, G) were clearly localized at the cleavage furrows between adjacent blastomeres in the absence of MC-LR. In contrast, $\beta$-catenin (Fig. 4B, D) and cadherins (Fig. 4F, H) were present only in the barely touched boundary between blastomeres. These results suggested that MC-LR perturbs the blastomere coherence by interfering with the localization of $\beta$-catenin and cadherin.

\section{Discussion}

The sensitivity to MC-LR exposure is variable in developmental stages and among fish species. The main cause of variability is the MC-LR uptake that is presumably mediated by bile acid transporters (Eriksson et al., 1990; Runnegar et al., 1991, 1995). To assay the toxicity of MC-LR during early embryonic development in zebrafish, we microinjected MC-LR directly into embryos and we observed that (1) MC-LR dose- and time-dependently increased the lethality of embryos. (2) MC-LR caused various embryo abnormalities, which suggested that MC-LR affected the phosphorylation status of upstream factors mastering the embryonic development and subsequent organogenesis. (3) MC-LR induced the loss of blastomere coherence by interfering the distributions of $\beta$-catenin and cadherins.

Zebrafish embryos and larvae were found to be insensitive to the toxicity of MC-LR or crude extracts of cyanobacteria. Although no acute effects were observed during embryonic development, a reduced survival rate and a retarded growth were reported in MC-LR and MC-RR preexposed larvae at 21 days of age (Oberemm et al., 1997, 1999). The lack of MC-LR toxicity in embryos is questionable, since the activities of MC-LR targets, PP1 and PP2A, have been shown to be critical for regulating cell cycle progression during embryonic development (Gotz et al., 2000). The most plausible explanation is the poor membrane permeability of microcystins in the absence of bile acid transporters. Jacquet et al. (2004) have nicely demonstrated the lethality of MC-LR on development of medaka fish embryos by microinjection. Using the similar approach and MC-LR dosages, we were able to demonstrate that MC-LR caused the death of embryos in a dose- and time-dependent manner (Fig. 2). We also observed a high rate in malformation in MC-LR-treated zebrafish embryos 

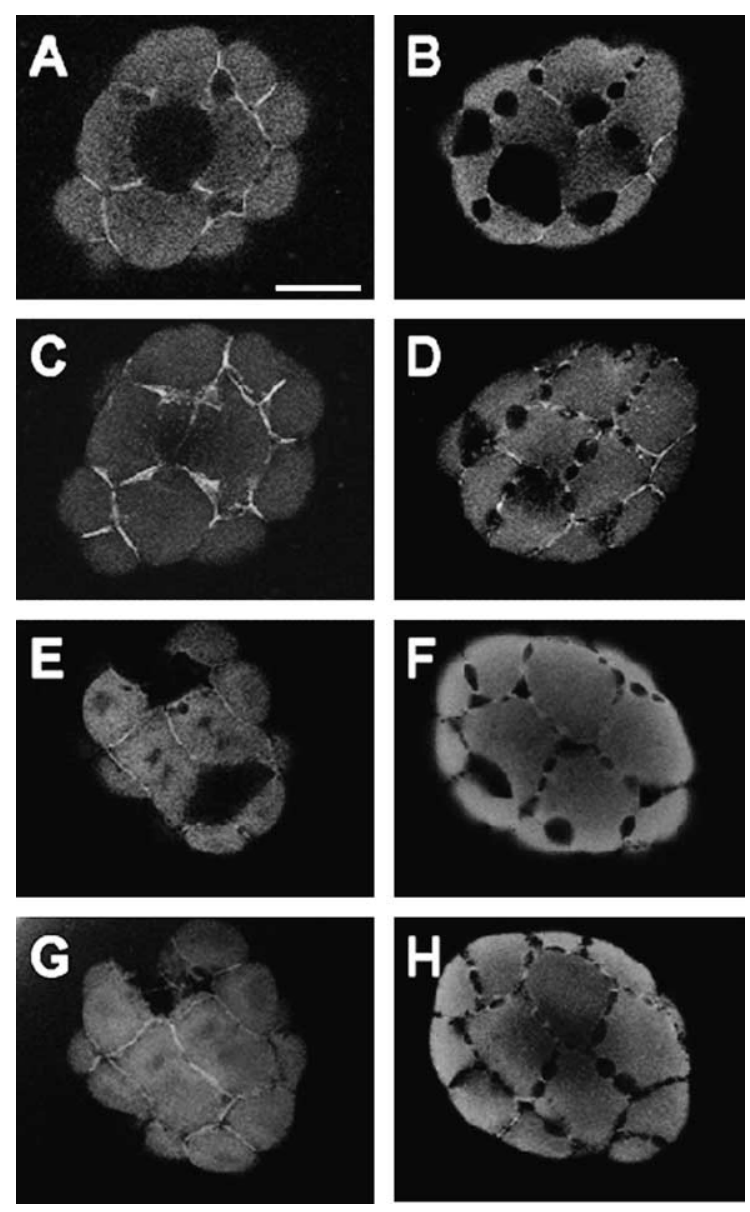

Fig. 4. MC-LR disturbs the distributions of $\beta$-catenin and cadherin. The MC-LR treated embryos were cultured until 16-cell stage, then fixed and stained with antibodies against $\beta$-catenin (A-D) or cadherin $(\mathrm{E}-\mathrm{H})$ and anti-rabbit FITC conjugated antibodies. Photographs of a single section of $3 \mu \mathrm{M}$ thick (A,B,E,F) and a 20 -section stacking graph $(\mathrm{C}, \mathrm{D}, \mathrm{G}, \mathrm{H})$ are shown. Both $\beta$-catenin or cadherin were clearly located between blastomeres in the controlinjected embryos (A,C,E,G), but the presence of both proteins were severely aborted in the presence of MC-LR (B,D,F,H). The scale bar represents $200 \mu \mathrm{M}$.

that is similar to reports in loach (Liu et al., 2002) and medaka (Jacquet et al., 2004). These results suggest that microinjection is a reliable bioassay system for embryonic toxicity of MC-LR. Furthermore, the effective MC-LR dosages (300-900 $\mathrm{nM}$ intracellular concentration) are comparable to the effective dosages utilized in a MC-LR microinjection study in medaka (Jacquet et al., 2004), however those dosages are much higher than the reported $\mathrm{IC}_{50}$ for PP1 and PP2A of about 0.1-1.0 nM (Honkanen et al., 1990). The possible explanation is the neutralization by detoxication enzymes like GST in embryos (Wiegand et al., 1999; Pietsch et al., 2001).

The interference of blastomeres coherence by MC-LR in early embryos is very interesting, since it implies the necessity of phosphorylation equilibrium for maintaining cell-cell adhesion. In cell and cell adhesion, cadherins play a central role in the assembly of adherens junctions (Yap et al., 1997). Knocking down E-cadherin by morpholino results in irregular orientation of cleavage planes between blastomeres, unequal cell sizes, reduced adhesion between blastomeres, and less compact embryos (Babb and Marrs, 2004), which is very similar to the phenotypes of MC-LRinjected embryos (Fig. 1). These results suggest that MC-LR may inhibit the cadherin-dependent cellular adhesions. Disturbance of cadherins distribution in cleavage furrows by MC-LR (Fig. 4) further strengthens the involvement of cadherins in MC-LR-induced toxicity.

The known targets of MC-LR are PP1 and PP2A. It would be interesting to know which phosphatase or both phosphatases is mediating the MC-LR-induced loss of blastomere adherence. The functions of classic cadherins are relying on its close association with catenins (Nagafuchi and Takeichi, 1988; Ozawa et al., 1989). Cadherin is also associated with $\beta$-catenin at the cleavage furrows in zebrafish embryos (Jesuthasan, 1998). Hyperphosphorylation of $\beta$-catenin on the serine-threonine residues induced by calyculin $\mathrm{A}$ and okadaic acid, known PP1 inhibitors, induced the loss of cellcell contacts in human epidermal cells (Serres et al., 1997). In this regards, MC-LR might inhibit PP1, which resulted in hyperphosphorylation of $\beta$-catenin, and the absence of both $\beta$-catenin and cadherin at the cleavage furrows (Fig. 4). Furthermore, PP2A has also been demonstrated to be critical in the regulation of $\beta$-catenin and E-cadherin via PP2A subunit $\mathrm{C} \alpha$ during embryonic development in mice (Gotz et al., 2000; Nunbhakdi-Craig et al., 2002). Therefore, both PP1 and PP2A may both involve in the MC-LR-mediated embryo malformation. Lastly, ezrin, a linker protein between the plasma membrane and the actin cytoskeleton, can regulate E-cadherin adherens junction assembly through rac1 activation (Pujuguet et al., 2003). More interestingly, phosphorylation of ezrin on threonine T567 was found to be critical for the compaction in early mouse embryos. It requires further investigation to see if the phosphorylation of zebrafish ezrin and subsequent activation of E-cadherin adherens junction assembly could be regulated by MC-LR mediated responses.

In conclusion, we had demonstrated that MC-LR induced the malformation and lethality of zebrafish embryos and larvae by disrupting the blastomere coherence. The loss of blastomere coherence by MC-LR is mediated presumably via the interference of $\beta$-catenin and cadherin adherens junction assembly. Our results clarify the controversial effects of MC-LR in zebrafish development. More importantly, we demonstrate that toxicity responses of embryos could be a useful bioassay system for detecting MC-LR.

\section{Acknowledgements}

We thank Yih-Min Chen for help in preparation of MC-LR. Fong-June Wu was a recipient of Undergraduate 
Research Grants (NSC91-2815-C-002-059-B) supported by National Science Council of Taiwan, R.O.C.

\section{References}

Babb, S.G., Marrs, J.A., 2004. E-cadherin regulates cell movements and tissue formation in early zebrafish embryos. Developmental Dynamics 230, 263-277.

Ding, W.X., Shen, H.M., Ong, C.N., 2000. Microcystic cyanobacteria extract induces cytoskeletal disruption and intracellular glutathione alteration in hepatocytes. Environmental Health Perspectives 108, 605-609.

Eriksson, J.E., Gronberg, L., Nygard, S., Slotte, J.P., Meriluoto, J.A., 1990. Hepatocellular uptake of 3H-dihydromicrocystin-LR, a cyclic peptide toxin. Biochemica et Biophysica Acta 1025, 60-66.

Fischer, W.J., Dietrich, D.R., 2000. Pathological and biochemical characterization of microcystin-induced hepatopancreas and kidney damage in carp (Cyprinus carpio). Toxicology and Applied Pharmacology 164, 73-81.

Frangez, R., Zuzek, M.C., Mrkun, J., Suput, D., Sedmak, B., Kosec, M., 2003. Microcystin-LR affects cytoskeleton and morphology of rabbit primary whole embryo cultured cells in vitro. Toxicon 41, 999-1005.

Gotz, J., Probst, A., Mistl, C., Nitsch, R.M., Ehler, E., 2000. Distinct role of protein phosphatase $2 \mathrm{~A}$ subunit $\mathrm{C}$ alpha in the regulation of E-cadherin and beta-catenin during development. Mechanisms of Development 93, 83-93.

Honkanen, R.E., Zwiller, J., Moore, R.E., Daily, S.L., Khatra, B.S., Dukelow, M., Boynton, A.L., 1990. Characterization of Microcystin-Lr, A Potent Inhibitor of Type-1 and Type-2A Protein Phosphatases. Journal of Biological Chemistry 265, 19401-19404.

Jacquet, C., Thermes, V., de Luze, A., Puiseux-Dao, S., Bernard, C., Joly, J.S., Bourrat, F., Edery, M., 2004. Effects of microcystinLR on development of medaka fish embryos (Oryzias latipes). Toxicon 43, 141-147.

Jesuthasan, S., 1998. Furrow-associated microtubule arrays are required for the cohesion of zebrafish blastomeres following cytokinesis. Journal of Cell Biology 111, 3695-3703.

Kimmel, C.B., Ballard, W.W., Kimmel, S.R., Ullmann, B., Schilling, T.F., 1995. Stages of embryonic-development of the zebrafish. Developmental Dynamics 203, 253-310.

Lee, T.H., Chen, Y.M., Chou, H.N., 1998. First report of microcystins in Taiwan. Toxicon 36, 247-255.

Liu, Y.D., Song, L.R., Li, X.Y., Liu, T.M., 2002. The toxic effects of microcystin-LR on embryo-larval and juvenile development of loach, Misguruns mizolepis Gunthe. Toxicon 40, 395-399.

Nagafuchi, A., Takeichi, M., 1988. Cell binding function of Ecadherin is regulated by the cytoplasmic domain. EMBO Journal 7, 3679-3684.

Nunbhakdi-Craig, V., Machleidt, T., Ogris, E., Bellotto, D., White, C.L., Sontag, E., 2002. Protein phosphatase 2A associates with and regulates atypical PKC and the epithelial tight junction complex. Journal of Cell Biology 158, 967-978.

Oberemm, A., Fastner, J., Steinberg, C.E.W., 1997. Effects of microcystin-LR and cyanobacterial crude extracts on embryolarval development of zebrafish (Danio rerio). Water Research 31, 2918-2921.
Oberemm, A., Becker, J., Codd, G.A., Steinberg, C., 1999. Effects of cyanobacterial toxins and aqueous crude extracts of cyanobacteria on the development of fish and amphibians. Environmental Toxicology 14, 77-88.

Ohta, T., Nishiwaki, R., Komori, A., Suganuma, M., Fujiki, H., 1992. Hyperphosphorylation of Cytokeratins 8 and 18 by Microcystin-LR, a new liver-tumor promoter, in primary cultured rat hepatocytes. Carcinogenesis 13, 2443-2447.

Ozawa, M., Baribault, H., Kemler, R., 1989. The cytoplasmic domain of the cell adhesion molecule uvomorulin associates with three independent proteins structurally related in different species. EMBO Journal 8, 1711-1717.

Pietsch, C., Wiegand, C., Ame, M.V., Nicklisch, A., Wunderlin, D., Pflugmacher, S., 2001. The effects of a cyanobacterial crude extract on different aquatic organisms: evidence for cyanobacterial toxin modulating factors. Environmental Toxicology 16, 535-542.

Pujuguet, P., del Maestro, L., Gautreau, A., Louvard, D., Arpin, M., 2003. Ezrin regulates E-cadherin-dependent adherens junction assembly through Rac1 activation. Molecular Biology of the Cell 14, 2181-2191.

Runnegar, M.T., Gerdes, R.G., Falconer, I.R., 1991. The uptake of the cyanobacterial hepatotoxin microcystin by isolated rat hepatocytes. Toxicon 29, 43-51.

Runnegar, M., Berndt, N., Kaplowitz, N., 1995. Microcystin uptake and inhibition of protein phosphatases: effects of chemoprotectants and self-inhibition in relation to known hepatic transporters. Toxicology and Applied Pharmacology 134, 264-272.

Runnegar, M., Wei, X., Zhang, L., HammAlvarez, S.F., 1997. Inhibition of protein phosphatase $2 \mathrm{~A}$ leads to enhanced phosphorylation of kinesin and cytoplasmic dynein and their associated proteins and to decreased microtubule-based vesicle motility in intact hepatocytes. Hepatology 26, 537.

Serres, M., Grangeasse, C., Haftek, M., Durocher, Y., Duclos, B., Schmitt, D., 1997. Hyperphosphorylation of beta-catenin on serine-threonine residues and loss of cell-cell contacts induced by calyculin A and okadaic acid in human epidermal cells. Experimental Cell Research 231, 163-172.

Tencalla, F., Dietrich, D., 1997. Biochemical characterization of microcystin toxicity in rainbow trout (Oncorhynchus mykiss). Toxicon 35, 583-595.

Tencalla, F.G., Dietrich, D.R., Schlatter, C., 1994. Toxicity of microcystis-aeruginosa peptide toxin to yearling rainbow-trout (Oncorhynchus-Mykiss). Aquatic Toxicology 30, 215-224.

Toivola, D.M., Goldman, R.D., Garrod, D.R., Eriksson, J.E., 1997. Protein phosphatases maintain the organization and structural interactions of hepatic keratin intermediate filaments. Journal of Cell Science 110, 23-33.

Watanabe, M.F., Harada, K.I., Carmicael, W.W., Fujiki, H., 1996. Toxic Microcystis. CRC press, Boca Raton, FL p. 262.

Wiegand, C., Pflugmacher, S., Oberemm, A., Meems, N., Beattie, K.A., Steinberg, C.E.W., Codd, G.A., 1999. Uptake and effects of microcystin-LR on detoxication enzymes of early life stages of the zebra fish (Danio rerio). Environmental Toxicology 14, 89-95.

Yap, A.S., Brieher, W.M., Gumbiner, B.M., 1997. Molecular and functional analysis of cadherin-based adherens junctions. Annual Review of Cell and Developmental Biology 13, 119-146. 